\title{
Photogated proton conductivity of ZIF-8 membranes co-modified with graphene quantum dots and polystyrene sulfonate
}

\author{
Shuaikang Fan, Shilin Wang, Xiaobin Wang, Zhuoyi Li, Xu Ma, Xinyi Wan, Shabab Hussain and \\ Xinsheng Peng
}

\begin{abstract}
Smart proton conductive metal-organic framework (MOF) membranes with dynamic remote control over proton conduction show high potential for use in advanced applications, such as sensors and bioprocesses. Here, we report a photoswitchable proton conductive ZIF-8 membrane by coencapsulating polystyrene sulfonate and graphene quantum dots into a ZIF-8 matrix (GQDs-PSS@ZIF-8) via a solidconfined conversion process. The proton conductivity of the GQDs-PSS@ZIF-8 membrane is 6.3 times higher than that of pristine ZIF-8 and can be reversibly switched by light due to photoluminescence quenching and the photothermal conversion effect, which converts light into heat. The local increase in temperature allows water molecules to escape from the porous channels, which cuts off the proton transport pathways and results in a decrease in proton conductivity. The proton conductivity is restored when the light is off owing to regaining water molecules, which act as proton carriers, from the surroundings. The GQDs-PSS@ZIF-8 membrane responds efficiently to light and exhibits an ON/OFF ratio of 12.8 . This photogated proton conduction in MOFs has potential for the development and application of MOF-based protonic solids in advanced photoelectric devices.
\end{abstract}

Keywords: ZIF-8, graphene quantum dots, photoswitchable proton conductivity, photoluminescence quenching, photothermal conversion

\section{INTRODUCTION}

Recently, solid-state proton conduction membranes have been increasingly studied due to their wide applications in devices, such as fuel cells, chemical or biological sensors, supercapacitors and information processing devices $[1,2]$. Based on their compositions, proton conducting solid membranes are chemically composed of inorganic oxides, ionic crystals, solid acid membranes, metalorganic frameworks (MOFs), porous coordination polymers (PCPs) and polymeric membranes $[3,4]$. MOFs are a subclass of highly porous and crystalline solid coordination compounds that are an assembly of metal clusters and organic linkers. MOFs possess versatile properties, such as well-defined porous structures, large surface areas and high structural diversity, which make MOFs suitable for use in gas storage, separation, catalysis and photoluminescence. The MOF structure can also be designed by pre- and post-synthetic methods to tailor the chemical stability and/or reactivity of the framework, enabling various functionalities for the utility mentioned above [58]. These appealing merits make MOFs exhibit promising potentials not only as host materials but also as excellent candidates for conducting ions, in particular protons, in the pores.

Proton conduction in MOFs has been widely studied in the past few years, with conductivities increasing from approximately $10^{-3}$ to $1 \mathrm{~S} \mathrm{~cm}^{-1}[4,9,10]$. Currently, many studies focus on achieving high proton conductivity via structural design, encapsulation of proton carriers, pore surface functionalization and so on in two distinct regimes-under hydrated conditions below $100^{\circ} \mathrm{C}$ and under anhydrous conditions above $100^{\circ} \mathrm{C}[5,11]$. However, the efficient and feasible control of proton conduction in response to external stimuli may be critical for a wide range of applications. To realize a dynamic switch of proton conduction in advanced devices, some external stimuli have been attempted, such as changing the heat, light, pressure, electric field and $\mathrm{pH}$ [12]. For example, pyranine was doped into a melt coordination polymer to

State Key Laboratory of Silicon Materials, School of Materials Science and Engineering, ERC of Membrane and Water Treatment, Ministry of Education, Zhejiang University, Hangzhou 310027, China

* Corresponding author (email: pengxinsheng@zju.edu.cn) 
achieve an optically reversible switch of proton conduction by light with a switch ratio of no more than 2 [13]. In most circumstances, light is regarded as a fast, reversible and usually noninvasive external signal to achieve remote control of proton conduction, attracting considerable attention.

Typically, one of the ways to switch proton conduction by light in MOFs is the introduction of light-responsive guest molecules to make use of their different characteristics, such as photoisomerization or photochromism [14]. The proton conductivity of photosensitive configuration molecule-decorated MOFs can be reversibly switched by ultraviolet (UV) light and restored by visible light or thermal relaxation $[15,16]$. However, the configuration change of photosensitive molecules is typically triggered by UV irradiation, which is potentially harmful to devices and operators. In addition, the configuration change may also require relatively large pores.

Another method to photomodulate proton conduction in MOFs relies on the interaction between stimuliresponsive materials and MOFs instead of the configuration changes of isomers. Some materials with photothermal conversion effects under light can alter proton conduction. However, the reversible switch of the proton conductivity of MOFs by doping photothermal nanomaterials is rarely reported. The only work on switching the proton conductivity of $\mathrm{Ag}$ nanoparticle-decorated DNA@MOFs was very recently realized and was based on the local plasma heating effect of Ag nanoparticles by our group [17]. In addition to noble metal nanoparticles, such as gold and silver [18,19], graphene quantum dots (GQDs) have been used as photothermal conversion materials for cancer therapy and other fields [20,21]. As an important member of the graphene family, GQDs have been increasingly studied because of their high photothermal conversion efficiency, excellent thermal and electrical conductivities, and low levels of toxicity [21]. Hence, it is expected that the introduction of GQDs in MOF membranes could help realize photoswitchable proton conductivity.

Zeolitic imidazolate framework-8 (ZIF-8) is a promising candidate as a host MOF for loading other additions since it is nontoxic and has excellent chemical and thermal stabilities in addition to the unique properties mentioned above [22-24]. Nevertheless, this framework is hydrophobic due to its organic linkers, leading to its low water uptake capacity and restricting the proton transport ability. Polystyrene sulfonate (PSS) is introduced as a foreign component to improve the water permeability of ZIF-8. PSS coordinates with ZIF-8 particles through the bonds between the $\mathrm{SO}^{3-}$ groups and $\mathrm{Zn}^{2+}$, making it more hydrophilic $[25,26]$. Moreover, the PSS polymer with ample sulfonate groups (approximately four sulfonate groups per nanometer) plays an important role as a proton carrier to promote proton conduction threading in ZIF-8 [27]. To the best of our knowledge, attempts have been made to introduce GQDs into porous ZIF-8 crystals to tune the luminescence property [28] or help kill cancer cells [19], but attempts to photoswitch proton conduction have seldom been investigated.

In this work, hybrid GQDs-PSS@ZIF-8 membranes were prepared by in situ incorporation of photothermal GQDs and hydrophilic PSS into ZIF-8, whose proton conduction can be altered by light. In the dark, proton transport mainly complies with the vehicular mechanism, and the proton conductivity of GQDs-PSS@ZIF-8 is approximately $3.49 \times 10^{-4} \mathrm{~S} \mathrm{~cm}^{-1}$ at $55^{\circ} \mathrm{C}$ and $95 \%$ relative humidity (RH); this result is 6.3 times higher than that of pristine ZIF-8. After turning on a light, the proton conductivity greatly decreases in a few minutes and then can be restored to its initial state when back in the dark; the highest switch factor is up to 12.8. When exposed to light, the photoluminescence (PL) quenching of ZIF-8 and photothermal conversion from GQDs increase the local temperature and dramatically heat the water molecules, resulting in the escape of water molecules from the proton transport paths. As soon as the light is off, water molecules are regained and consequently rebuild the proton conductive channels with PSS by reforming hydrogen bonds. Thus, a reversible switch of the proton conductive process is achieved. This one-step approach to construct a photoswitchable MOF membrane is efficient, convenient and ecofriendly. The easy control mode under a single light source endows the GQDs-PSS@ZIF-8 membranes with promising potential for use in dynamic remote-controlled devices and applications.

\section{EXPERIMENTAL SECTION}

\section{Materials and chemicals}

Zinc nitrate $\left(\mathrm{Zn}\left(\mathrm{NO}_{3}\right)_{2} \cdot 6 \mathrm{H}_{2} \mathrm{O}\right)$ and 2-aminoethanol $\left(\mathrm{NH}_{2}-\mathrm{CH}_{2} \mathrm{CH}_{2} \mathrm{OH}\right)$ were purchased from Acros Chemicals. 2-Methylimidazole ( $\left.\mathrm{Hmim}, \mathrm{C}_{4} \mathrm{H}_{6} \mathrm{~N}_{2}\right)$ was purchased from Sigma-Aldrich. PSS and aqueous dispersion of GQDs $\left(1 \mathrm{mg} \mathrm{mL}^{-1}\right)$ were purchased from Aladdin. Ethanol was purchased from Sinopharm Chemical Reagent Co. Ltd. All reagents were used without further purification. The membrane used to filter was polycarbonate (PC) membranes (Whatman) with a pore diameter of $200 \mathrm{~nm}$ and an effective diameter of $20 \mathrm{~mm}$. Ultrapure 
water of 18.2 $\mathrm{M} \Omega$ was produced by a Millipore direct-Q system throughout all experiments.

\section{Synthesis of GQDs-PSS@ZIF-8 membrane}

The zinc hydroxide nanostrand $(\mathrm{ZHN})$ solution was prepared by mixing $4 \times 10^{-3} \mathrm{~mol} \mathrm{~L}^{-1}$ zinc nitrate water/ ethanol solution $(3: 2)$ with the same volume of $1.6 \times$ $10^{-3} \mathrm{~mol} \mathrm{~L}^{-1}$ 2-aminoethanol water/ethanol solution (3:2) at room temperature and aging for $30 \mathrm{~min}$ [29]. Then, $10 \mathrm{~mL}$ of ZHN solution was mixed with PSS solution $\left(1 \mathrm{mg} \mathrm{mL}^{-1}\right)$ and aqueous dispersion of GQDs $\left(0.1 \mathrm{mg} \mathrm{mL}^{-1}\right)$ of different volumes as required. The GQDs-PSS@ZHN thin film was obtained by filtering the mixed solution on PC membrane. Afterwards, the GQDsPSS@ZHN thin film on PC membrane was transferred to a piece of clean glass wetted by ethanol and kept in ethanol for a few minutes. The membrane was dried at room temperature for at least $30 \mathrm{~min}$. Finally, the GQDsPSS@ZIF-8 membrane was synthesized by immersing the GQDs-PSS@ZHNs film into $25 \times 10^{-3} \mathrm{~mol} \mathrm{~L}^{-1} \mathrm{Hmim}$ water/ethanol solution $(4: 1)$ at room temperature for $24 \mathrm{~h}$. After reacting, the membrane was washed with water/ethanol solution (4:1) at least three times and dried at room temperature.

\section{Characterization}

The X-ray diffraction (XRD) patterns of the as-prepared membranes were characterized by X'Pert PRO (PANalytical, The Netherlands) instrument with $\mathrm{Cu} \mathrm{Ka}$ radiation at $0.02^{\circ}$ step at room temperature. The morphologies of the resulting samples were characterized by scanning electron microscopy (SEM) (Hitachi S-4800/SU-70) equipped with $\mathrm{X}$-ray energy dispersive analysis and transmission electron microscopy (TEM) (HT-7700) equipped with X-ray energy dispersive analysis (Oxford). The Fourier transform infrared (FTIR) spectra were carried out on FTIR TENSOR 27 equipment using $\mathrm{KBr}$ pellets in the wavenumber range of $400-4000 \mathrm{~cm}^{-1}$. The UV-visible (UV-vis) absorption spectra were measured by a UV-vis spectrophotometer (Shimadzu UV-3600). The particle size distribution and zeta potential were measured by Zetasizer Nano-ZS (Malvern). The $\mathrm{N}_{2}$ sorption isotherms were measured at $77 \mathrm{~K}$ by Micromeritics instrument (3Flex). Raman spectroscopy measurements were carried out at room temperature on a Raman spectrophotometer (LabRAM HR Evolution) using a 532-nm laser. X-ray photoelectron spectroscopy (XPS, AXIS SUPRA, Krato) was used to confirm the existence of PSS. The PL spectra were recorded using a spectrophotometer (Edinburgh Instruments FLS920) with a 325-nm laser.

\section{Conductivity measurement}

The electrochemical impedance measurements were carried out by a CHI 660D electrochemical workstation in the frequency range from $1 \mathrm{MHz}$ to $100 \mathrm{~Hz}$ at alternating current (AC) amplitude of $0.1 \mathrm{~V}$. Two Ag electrodes were evaporated onto the surface of the membrane on glass substrate by ZHD-300S film preparation system (BEIJING TECHNOL Co. Ltd.) with current of $65 \mathrm{~A}$ for $30 \mathrm{~min}$. Before the electrochemical impedance test, the membranes were placed in certain temperature and humidity conditions more than $2 \mathrm{~h}$ to be stable. The temperature and humidity were controlled by the BC1300 programmable temperature and humidity controller (Shanghai YIHENG). The light source (PLS-SXE300D/ UV, PerfectLight, the visible light range covered) was placed outside the glass windows of the temperature and humidity controller at a fixed distance to do the electrochemical impedance test under light. The power density of light at the sample position was around $50 \mathrm{~mW} \mathrm{~cm}^{-2}$ measured by PLS-MW2000 (PerfectLight). The infrared filter (BRPASS) and UV filter (UVCUT420) were bought from Beijing Perfectlight Technology Co. Ltd.

The proton conductivity $(\sigma)$ was calculated according to the physical dimensions of the samples based on the following equation:

$\sigma=\frac{L}{R S}$

where $L$ is the channel length (here is $300 \mu \mathrm{m}$ ), $R$ is the resistance value calculated from electrochemical impedance spectra (using $R$ s (CPE-Rp) model to fit the data), and $S$ is the cross-section area of the flow transportation surface (here is $3 \mathrm{~mm}$ (width) $\times 1 \mu \mathrm{m}$ (thickness)) for GQDs-PSS@ZIF-8 membranes.

In order to explore the effect of light on proton conductivity of the membranes, a Xenon lamp was placed outside of the programmable controller at a constant distance with a power density of $50 \mathrm{~mW} \mathrm{~cm}^{-2}$ (Fig. S1). The proton conductivity ON/OFF ratio is defined as the value of proton conductivity in dark to that under light in steady-state situation and used to estimate the photoswitchable performance.

\section{RESULTS AND DISCUSSION}

The GQDs-PSS@ZIF-8 membrane was synthesized through a solid confinement conversion process [29] from a thin film of ZHNs mixed with PSS solution and an 
aqueous dispersion of GQDs at room temperature (Scheme 1). The negatively charged PSS [27] and GQDs (zeta potential of $-7.38 \mathrm{mV}$, Fig. S2) were adsorbed tightly on the surface of the highly positively charged ZHNs through electrostatic interactions before the ZHNs were transformed into ZIF-8 [30,31]. When immersed in a Hmim water/ethanol solution, the ZHNs on the top grew into a continuous ZIF-8 layer covering the surface of GQDs-PSS@ZHNs film. The already formed dense ZIF-8 layer prevented the PSS and GQDs from escaping from the composite film into solution, resulting in a nice GQDs-PSS@ZIF-8 membrane with PSS and GQDs well encapsulated in the ZIF-8 crystal matrix.

Before using GQDs, different amounts of PSS (0.01, 0.1, and $0.2 \mathrm{mg}$ ) were added to an equal volume of $\mathrm{ZHN}$ solution to investigate the impact of the PSS amount on the morphology and integrity of the ZIF- 8 membrane. The membranes were named 1PSS@ZIF-8, 2PSS@ZIF-8 and 3PSS@ZIF-8, with PSS to ZIF-8 weight ratios of 1.1\%, $11 \%$ and $22 \%$ (Table $S 1$ ), respectively. A series of GQDsPSS@ZIF-8 membranes with different GQD contents were synthesized based on 2PSS@ZIF-8 using 0.01, 0.05,
$0.1,0.2$ and $0.3 \mathrm{mg}$ GQDs, leading to weight ratios of GQDs to ZIF-8 of $1.1 \%, 5.5 \%, 11 \%, 22 \%$ and $33 \%$, respectively; these samples are denoted as 1GQDsPSS@ZIF-8, 2GQDs-PSS@ZIF-8, 3GQDs-PSS@ZIF-8, 4GQDs-PSS@ZIF-8 and 5GQDs-PSS@ZIF-8 (Table S1), respectively.

Fig. la-f show the surface and cross-sectional SEM images of the as-synthesized ZIF-8 and 2GQDsPSS@ZIF-8 membranes. It is clear that the ZIF-8 and 2GQDs-PSS@ZIF-8 membranes have similar morpholo-

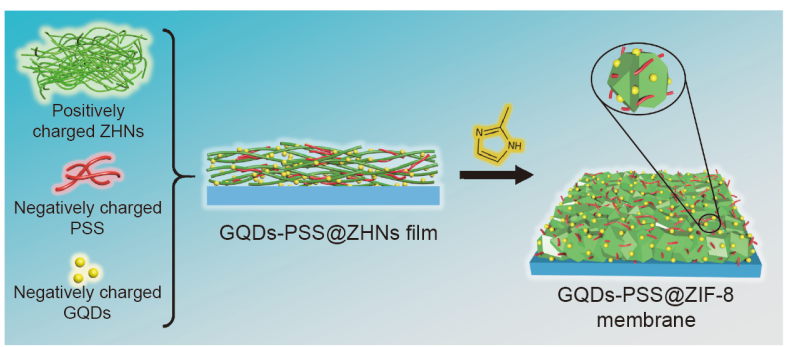

Scheme 1 Illustration of the synthesis process of GQDs-PSS@ZIF-8 membrane.
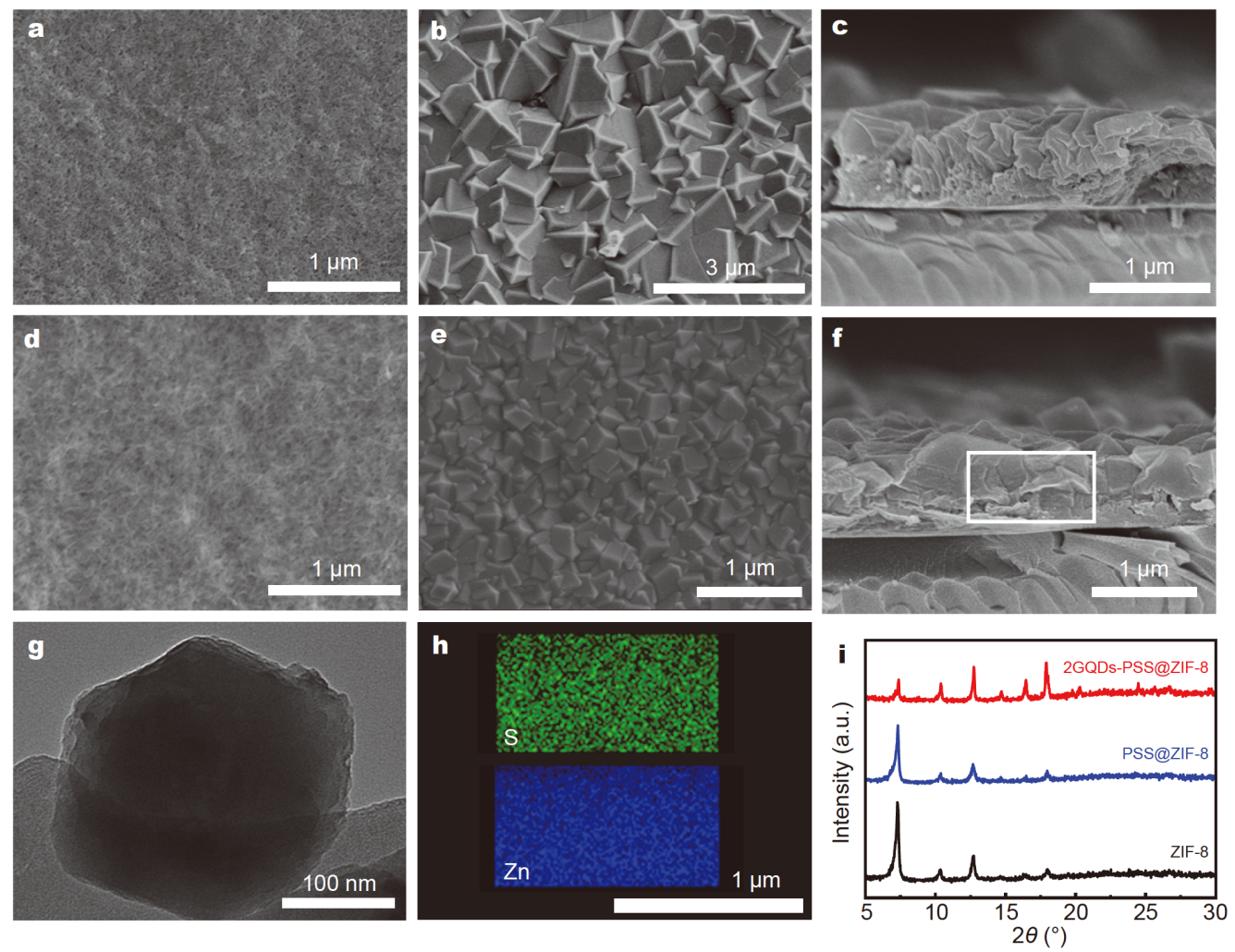

Figure 1 (a) SEM image of the ZHNs. (b) Surface and (c) cross-sectional SEM images of the ZIF-8 membrane. (d) SEM image of 2GQD-PSS@ZHNs. (e) Surface and (f) cross-sectional SEM images of 2GQDs-PSS@ZIF-8 membrane. (g) TEM image of the 2GQDs-PSS@ZIF-8 membrane. (h) S (green) and Zn (blue) EDS mapping images of the zone marked in (f). (i) XRD patterns of the ZIF-8, PSS@ZIF-8 and 2GQDs-PSS@ZIF-8 membranes. 
gies with continuous well intergrown ZIF-8 crystals. PSS can enhance the proton transport in ZIF-8 pores, but too much PSS harms the continuity and integrity of the membrane, creating an incompact surface (Fig. S3a-c). The surface of 3PSS@ZIF-8 is not as good as the other two apparently. As shown in Fig. 1c, $\mathrm{f}$, the thicknesses of ZIF-8 and 2GQDs-PSS@ZIF-8 are both approximately $1 \mu \mathrm{m}$, which is used to calculate proton conductivity. The TEM image (Fig. 1g) shows that the as-prepared 2GQDsPSS@ZIF-8 nanocrystals are rhombic dodecahedron particles with a size of approximately $300 \mathrm{~nm}$. The crosssectional energy dispersive spectroscopy (EDS) mapping images (Fig. 1h) and TEM mapping images (Fig. S4) of 2GQDs-PSS@ZIF-8 show a uniform distribution of S and $\mathrm{Zn}$, demonstrating that PSS is encapsulated in the pores of ZIF-8 and uniformly distributed in the membrane.

The XRD patterns (Fig. 1i and Fig. S5a) show that the ZIF-8 phase successfully forms with sharp diffraction peaks in GQDs-PSS@ZIF-8 membranes and that the GQDs and PSS do not affect the formation of ZIF-8 crystals. In the FTIR spectra (Fig. 2a and Fig. S5b), a wide characteristic peak from 1191 to $1250 \mathrm{~cm}^{-1}$, which represents the sulfonate groups in the pure PSS powders, appears in PSS@ZIF-8 and 2GQDs-PSS@ZIF-8 but is absent in the pristine ZIF-8 membrane. The peak at $1039 \mathrm{~cm}^{-1}$ existing in PSS@ZIF-8 and 2GQDs-PSS@ZIF-8 is different from the $1045 \mathrm{~cm}^{-1}$ peak in the ZIF-8 membrane, and this shift can be attributed to the interaction of $\mathrm{Zn}^{2+}$ with part of the sulfonate groups in PSS [26]. The $\mathrm{N}_{2}$ sorption isotherms of the ZIF-8 and PSS@ZIF-8 membranes (Fig. S6a) were measured to determine the surface area and check the encapsulation of PSS on ZIF- 8 crystals. The specific surface area of PSS@ZIF-8 $\left(962.4 \mathrm{~m}^{2} \mathrm{~g}^{-1}\right)$ is lower than $1326.5 \mathrm{~m}^{2} \mathrm{~g}^{-1}$ of ZIF-8 because of the encapsulation of PSS in the membrane. Raman spectroscopy was used to prove the incorporation of GQDs. The Raman peaks of GQDs are observed in 2GQDs-PSS@ZIF-8 due to the encapsulation of GQDs inside ZIF-8 (Fig. S6b). XPS results show the existence of S in PSS@ZIF-8 and 2GQDs-PSS@ZIF-8 (Fig. S7), further confirming the successful incorporation of PSS in ZIF-8. The PL spectra of the solutions with different dissolved GQDs-PSS@ZIF8 membranes (Fig. S8a) show that the largest peak of GQDs is clearly observed at approximately $495 \mathrm{~nm}$, matching the aqueous dispersion of GQDs (Fig. S8b). From the above results, it is confirmed that PSS and GQDs have been successfully encapsulated into ZIF-8 crystals.

According to the UV-vis absorption spectra (Fig. 2b, Fig. S8c),2GQDs-PSS@ZIF-8 absorbs more UV-vis light than the ZIF-8 and PSS@ZIF-8 membranes due to the GQDs absorbing a considerable amount of short-wavelength light. The increasing absorption of PSS@ZIF-8 may be ascribed to the benzene ring and sulfonate groups. Hence, GQDs-PSS@ZIF-8 membranes show better absorption, especially UV light, because of their GQD contents. The PL spectra of the as-prepared membranes (Fig. 2c) depict that the fluorescence intensity of the ZIF8 membrane at $395 \mathrm{~nm}$ is stronger than those of the PSS@ZIF-8 and GQD-PSS@ZIF-8 membranes, and no obvious PL peaks of GQDs appear (Fig. S8b). ZIF-8 has blue luminescence owing to organic Hmim linkers coordinated with the central transition metal $\left(\mathrm{Zn}, \mathrm{d}^{10}\right.$ configuration) ions; this arrangement makes the potential charge/energy transfer between $\mathrm{Hmim}$ and $\mathrm{Zn}$ ions possible, and the peak position relates to the excitation wavelength $[23,28,32]$. By adding PSS and GQDs, the fluorescence of ZIF-8 is quenched, and the addition of more GQDs in GQDs-PSS@ZIF-8 membranes result in a lower luminescence intensity because of the interaction between ZIF-8 and GQDs. The decrease in PL intensity indicates that the loss of optical energy is released in the form of heat, which is the most important process to reversibly switch the proton conductivity by light in
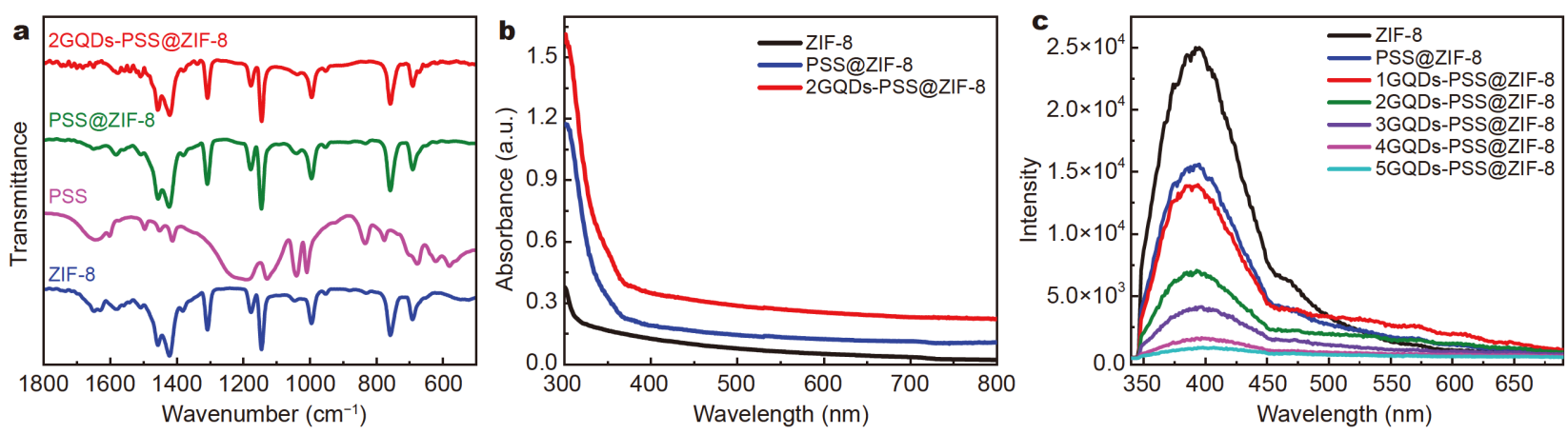

Figure 2 (a) FTIR and (b) UV-vis absorption spectra of the samples. (c) PL spectra of the membranes excited at $325 \mathrm{~nm}$. 
porous MOF membranes.

The proton conductivities of the pristine ZIF-8, PSS@ZIF-8 and GQDs-PSS@ZIF-8 membranes were investigated by electrochemical impedance. The samples were kept in a temperature and humidity controller and measured at a constant temperature of $55^{\circ} \mathrm{C}$ and $95 \% \mathrm{RH}$. If not specifically mentioned, the proton conductivity is measured in the dark. In Fig. 3a, the proton conductivity $\left(1.45 \times 10^{-3} \mathrm{~S} \mathrm{~cm}^{-1}\right)$ of PSS@ZIF-8 is 25 times higher than that $\left(5.52 \times 10^{-5} \mathrm{~S} \mathrm{~cm}^{-1}\right)$ of pristine ZIF-8 due to the numerous sulfonate groups in PSS@ZIF-8. The proton conductivity of 1PSS@ZIF-8 $\left(1.60 \times 10^{-4} \mathrm{~S} \mathrm{~cm}^{-1}\right)$ is approximately one-tenth of 2PSS@ZIF- 8 . The membrane with relatively higher proton conductivity should be better according to our previous work $[2,17]$. Hence, the series of GQDs-PSS@ZIF-8 membranes were synthesized based on 2PSS@ZIF-8. In contrast, GQDs-PSS@ZIF-8 membranes exhibit lower proton conductivity, decreasing from $5.66 \times 10^{-4} \mathrm{~S} \mathrm{~cm}^{-1}$ for 1GQDs-PSS@ZIF-8 to $3.49 \times$ $10^{-4} \mathrm{~S} \mathrm{~cm}^{-1}$ for 2GQDs-PSS@ZIF-8, $7.68 \times 10^{-5} \mathrm{~S} \mathrm{~cm}^{-1}$ for 3GQDs-PSS@ZIF-8, $5.94 \times 10^{-6} \mathrm{~S} \mathrm{~cm}^{-1}$ for 4GQDsPSS@ZIF-8 and $7.05 \times 10^{-6} \mathrm{~S} \mathrm{~cm}^{-1}$ for 5GQDs-PSS@ZIF-
8. The proton conductivity decreases with an increasing amount of GQDs in the GQDs-PSS@ZIF-8 membranes. However, the proton conductivity of 2GQDs-PSS@ZIF-8 is 6.3 times higher than that of pristine ZIF-8, which is still a remarkable enhancement. Although PSS has provided sulfonate groups and introduces more water molecules to speed up proton transport, the large GQD particles may lead to discontinuities and defects in the membranes and the distortion of PSS chains, partly blocking the proton transport paths and ultimately resulting in lower proton conductivity.

The influences of temperature and $\mathrm{RH}$ on the proton conductivities of the resulting membranes are shown in Fig. 3b-d. The impedance of 2GQDs-PSS@ZIF-8 decreases, and the proton conductivity increases from 1.00 $\times 10^{-4}$ to $2.64 \times 10^{-3} \mathrm{~S} \mathrm{~cm}^{-1}$ with increasing temperature in the range from 45 to $85^{\circ} \mathrm{C}$ and $95 \% \mathrm{RH}$. Under stable conditions, more water molecules in the membranes become active by accepting energy from the environment, and protons can be transported more easily from one place to another site. The transport mechanism will be discussed later. Moreover, when the temperature is $55^{\circ} \mathrm{C}$,
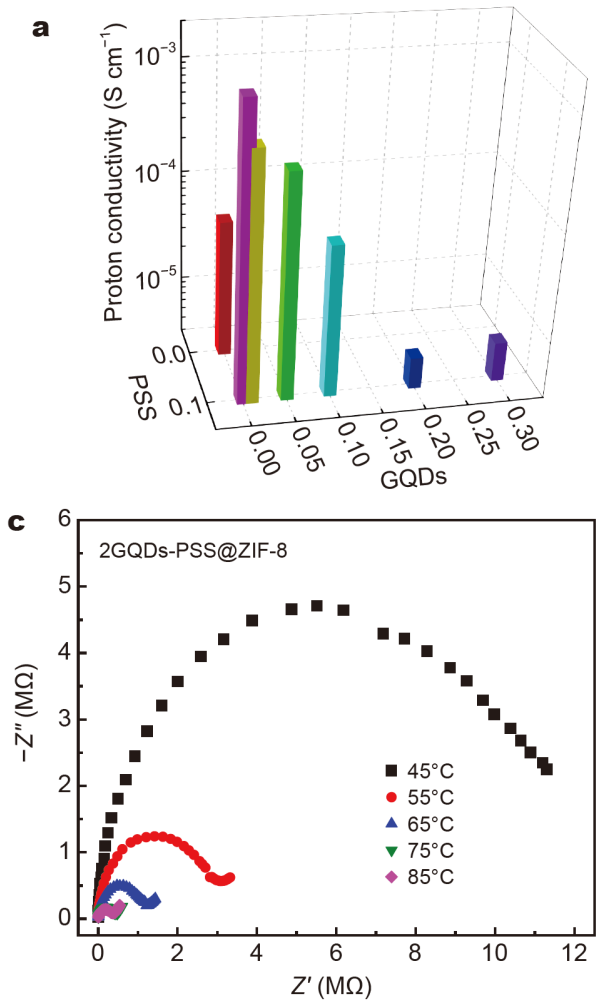
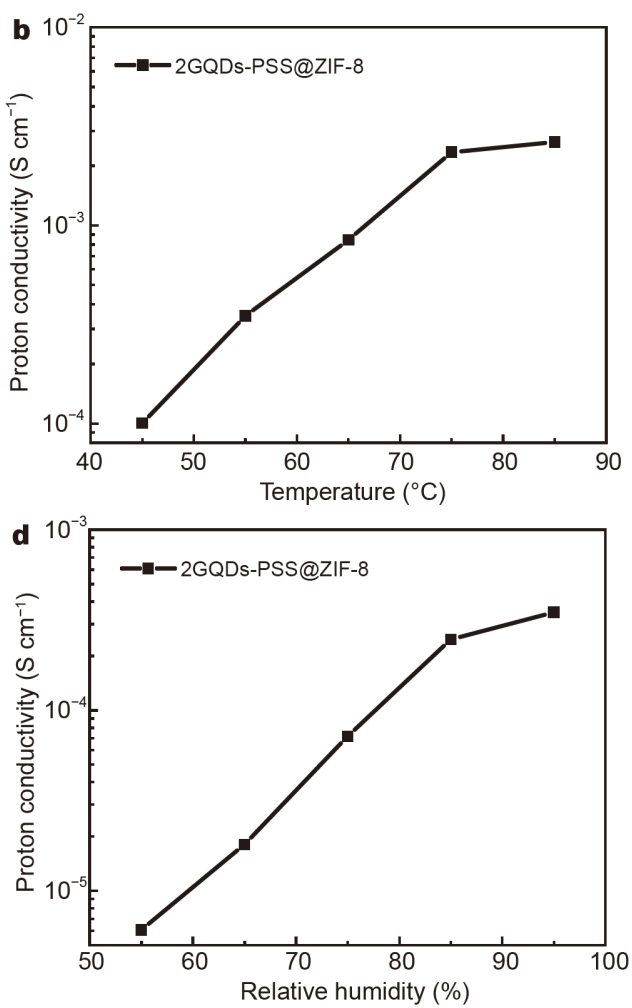

Figure 3 (a) Proton conductivities of ZIF-8, PSS@ZIF-8 and GQDs-PSS@ZIF-8 with different GQD contents at 55 $\mathrm{C}$ and 95\% RH. (b) Proton conductivities of 2GQDs-PSS@ZIF-8 at different temperatures and 95\% RH. (c) Nyquist plots of 2GQDs-PSS@ZIF-8 corresponding to (b). (d) Proton conductivities of 2GQDs-PSS@ZIF-8 at different humidities and $55^{\circ} \mathrm{C}$. 
the proton conductivity of 2GQDs-PSS@ZIF-8 increases from $6.07 \times 10^{-6}$ to $3.49 \times 10^{-4} \mathrm{~S} \mathrm{~cm}^{-1}$ when the humidity is changed from $55 \%$ to $95 \%$ RH, as shown in Fig. 3d and Fig. S8d. Apparently, the proton conductivity has a strong relation with $\mathrm{RH}$ because the presence of more water molecules inside the porous channels can promote the formation of the hydrogen-bond network and introduce more proton carriers, thus facilitating proton transport.

Furthermore, the proton conductivities of the samples at different temperatures were measured to investigate the proton conduction mechanism. As shown in Fig. 4a and Fig. S8e, proton transports in the ZIF-8, PSS@ZIF-8 and GQDs-PSS@ZIF-8 membranes follow Arrhenius-like behavior with activation energies ranging from 0.43 to $1.03 \mathrm{eV}$ under $95 \% \mathrm{RH}$. The activation energy of ZIF-8 is $0.43 \mathrm{eV}$, close to the value for the Grotthuss mechanism $(\leq 0.4 \mathrm{eV})$, indicating that proton conduction in the ZIF-8 membrane follows the Grotthuss mechanism through the hydrogen-bond network formed by water in the channels [33]. After PSS and GQDs are incorporated in the membranes, the activation energy values are higher than $0.43 \mathrm{eV}$, being in the range of the vehicle mechanism $[34,35]$. This result suggests that many water molecules and sulfonate groups in the PSS@ZIF-8 and GQDPSS@ZIF-8 membranes have the possibility to accept protons and transport protons through direct self-diffusion in the membranes. Additionally, provided that hydrogen-bond cleavage requires a low energy value in the range of $2-3 \mathrm{kcal} \mathrm{mol}^{-1}(\sim 0.11 \mathrm{eV})$ [5], the formation of the hydrogen-bond network among sulfonate groups and water molecules is easy, but the Grotthuss mechanism is no longer dominant. Considering the pore cavities (11.6 $\AA$ ) and small apertures $(3.4 \AA)$ of the ZIF-8 structure [25], PSS threaded in ZIF- 8 narrows the apertures, making it more difficult for water molecules to diffuse through the membrane with an elevated activation energy [10]. Nevertheless, PSS helps increase the hydrophilicity of ZIF-8 to attract water molecules into the pores, bringing more proton carriers into the membrane. Accordingly, this mechanism accounts for the large difference between pristine ZIF-8 and the PSS@ZIF-8 and GQDs-PSS@ZIF-8 membranes. GQDs have a larger size (5-6 nm, Fig. S9) than the cavity of ZIF-8, making it impossible for them to embed into ZIF-8. However, GQDs can be easily encapsulated in the ZIF-8 crystalline membrane to block proton conduction along the PSS

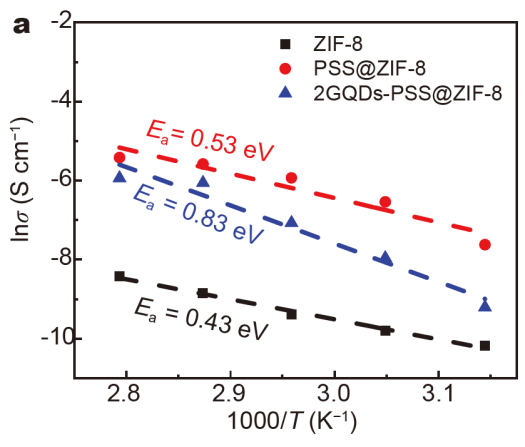

d

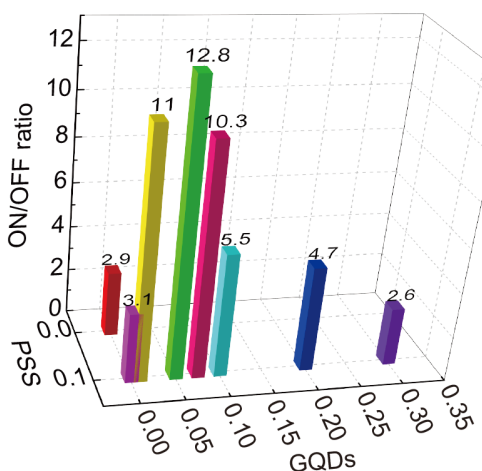

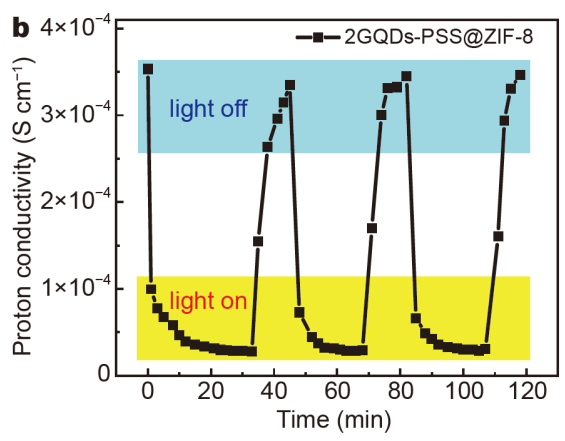

e

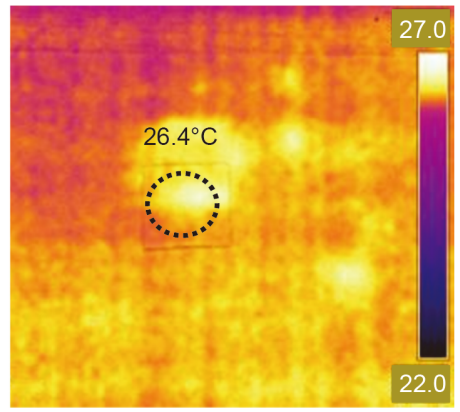

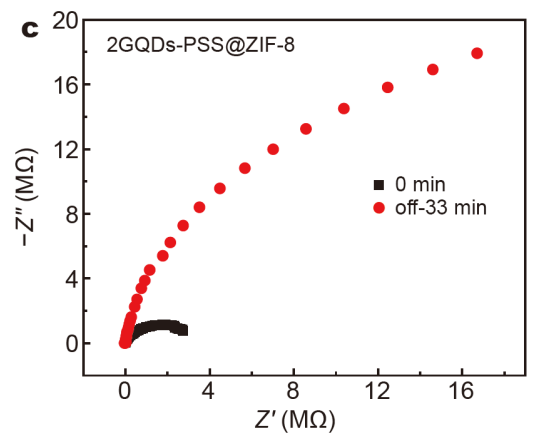

f

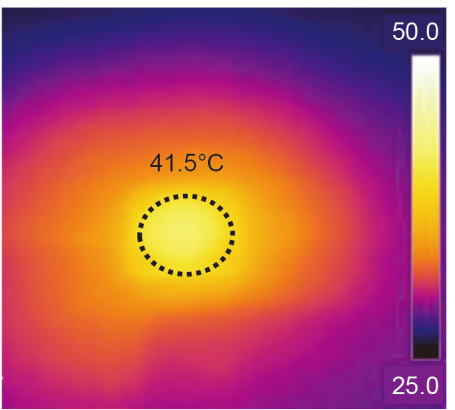

Figure 4 (a) Arrhenius plots and corresponding activation energies of ZIF-8, PSS@ZIF-8 and 2GQDs-PSS@ZIF-8 at 95\% RH. (b) Proton conductivities of 2GQDs-PSS@ZIF-8 with the light off (blue region) and on (yellow region) at $55^{\circ} \mathrm{C}$ and $95 \%$ RH. (c) Corresponding Nyquist plots of (b). (d) ON/OFF ratios of the ZIF-8, PSS@ZIF-8 and GQDs-PSS@ZIF-8 membranes with different GQD contents at $55^{\circ} \mathrm{C}$ and $95 \% \mathrm{RH}$. Infrared camera photographs of 2GQDs-PSS@ZIF-8 (e) before and (f) after exposure to the Xenon lamp for $10 \mathrm{~min}$ at an optical power density of $100 \mathrm{~mW} \mathrm{~cm}^{-2}$. 
chains, and affect the intergrowth of ZIF-8 crystal, thereby increasing the resistance. Consequently, and the proton conductivities of GQDs-PSS@ZIF-8 membranes are lower than that of PSS@ZIF-8, and 1GQDs-PSS@ZIF8 has the highest conductivity among the GQDsPSS@ZIF-8 membranes.

According to previous work on photoswitchable proton conduction in MOF membranes, a relatively low temperature is beneficial to photoswitching performance and highlights the effect of heat coming from light $[2,17]$. A high proton conductivity can be achieved at a high humidity. Hence, a temperature of $55^{\circ} \mathrm{C}$ and a constant humidity of $95 \%$ RH are applied in this study. Fig. 4b, c and Figs S10 and S11 describe the behaviors of the membranes in light and dark in a consecutive process. Turning off the light, the proton conductivity of 2GQDsPSS@ZIF-8 reaches $3.53 \times 10^{-4} \mathrm{~S} \mathrm{~cm}^{-1}$ and greatly decreases to $2.76 \times 10^{-5} \mathrm{~S} \mathrm{~cm}^{-1}$ once exposed to light, resulting in an ON/OFF ratio of 12.8. The speed of the photoswitch is calculated accordingly. The decrease of proton conductivity $(80 \%)$ happened within $1 \mathrm{~min}$ in 2GQDs-PSS@ZIF-8. Hence, the membrane responds rapidly in a few minutes and is restored to its initial state in the dark, showing good photoswitching performance. The corresponding Nyquist plots of 2GQDs-PSS@ZIF-8 (Fig. 4b, c) after consecutive switching cycles indicate its good cycling and recovery performances. 6GQDsPSS@ZIF-8 was prepared later to help find the highest ON/OFF ratio (Fig. S12). The ZIF-8 and PSS@ZIF-8 membranes have low ON/OFF ratios of 2.9 and 3.1, respectively, compared with 2GQDs-PSS@ZIF-8 (Fig. 4d). As the GQD content increases, the ON/OFF ratio first increases from 11 for 1GQDs-PSS@ZIF-8 to 12.8 for 2GQDs-PSS@ZIF-8 and then decreases to 2.6 for 5GQDsPSS@ZIF-8. Different from 2GQDs-PSS@ZIF-8, whose proton conductivity fluctuates in a large range, the ZIF- 8 and PSS@ZIF-8 membranes, lacking GQDs, exhibit a smaller change under alternating light and dark regardless of their own specific proton conductivities. To some extent, GQDs do increase the sensitivity to light in some membranes, but this does not mean that more GQDs provide a higher ON/OFF ratio. The low ON/OFF ratio may be due to the GQD barrier effect, which makes it difficult to smoothly transport protons. There is a tradeoff between proton conductivity and the ON/OFF ratio. Both of them should be taken into consideration. On the one hand, the photoswitchable proton conduction controlled by light depends on GQDs. On the other hand, GQDs have a partial disadvantage in regard to proton conductivity. That is why 2GQDs-PSS@ZIF-8 has the highest ON/OFF ratio with relatively high proton conductivity. To study the effect of light at different wavelengths on the photoswitching performance, consecutive switching cycles under light and dark conditions using an infrared filter and a UV filter were measured (Fig. S13). With the infrared filter, the ON/OFF ratio of 2GQDsPSS@ZIF-8 decreases slightly to 11.4. However, the ON/ OFF ratio (6.1) with the UV filter is less than half of the highest switch factor (12.8) without the filter. This result fits with the UV-vis absorption spectra and indicates that UV and visible light play a dominant role in the photoswitching process.

Scheme 2 explains the proton conducting mechanism in response to light. The PSS chains and plentiful water molecules provide proton transport highways in 2GQDsPSS@ZIF-8, similar to the bridge across GQDs built in the ZIF-8 frameworks in the dark. When exposed to light, GQDs convert optical energy into heat by quenching the fluorescence of ZIF-8 and the photothermal conversion effect. The water molecules distributed in the proton conductive channels are removed. Then, the proton transport paths are narrowed or even cut off in some local high-temperature zones, leading to low proton conductivity. This mechanism can be inferred from Fig. S14, in which the wide peak at $3450 \mathrm{~cm}^{-1}$ in the dark, which belongs to the stretching vibration of $\mathrm{O}-\mathrm{H}$, is not as obvious as that after light irradiation in PSS@ZIF-8 and 2GQDs-PSS@ZIF-8, indicating the removal of water molecules. Moreover, the decrease in the number of water molecules in ZIF-8 and PSS@ZIF-8 is not as much as that in 2GQDs-PSS@ZIF-8 (Table S2). This result also explains the highest ON/OFF ratio of 2GQDs-PSS@ZIF-8.

The energy conversion used to switch the proton conductivity is probably due to the following three reasons. First, the fluorescence of ZIF-8 is quenched by PSS and GQDs in the GQDs-PSS@ZIF-8 membranes, causing photothermal conversion. The fluorescence of PSS is very weak compared with that of GQDs (Fig. S8b). However, PSS, which coordinates with ZIF-8 particles through the bonding between the sulfonate groups and $\mathrm{Zn}^{2+}$, may strengthen the electron-withdrawing capability of sulfonate groups, possibly leading to the lower fluorescence intensity of GQDs-PSS@ZIF-8 membranes [28]. It has been reported that GQDs have good electrical conductivity and high electron mobility and easily accept electrons. These merits can facilitate electron transfer in membranes [36,37]. Therefore, the electrons excited in ZIF-8 are likely to transfer from the central transition metal ions to the GQDs and relax in the GQDs to generate thermal energy at the cost of optical energy. Second, 


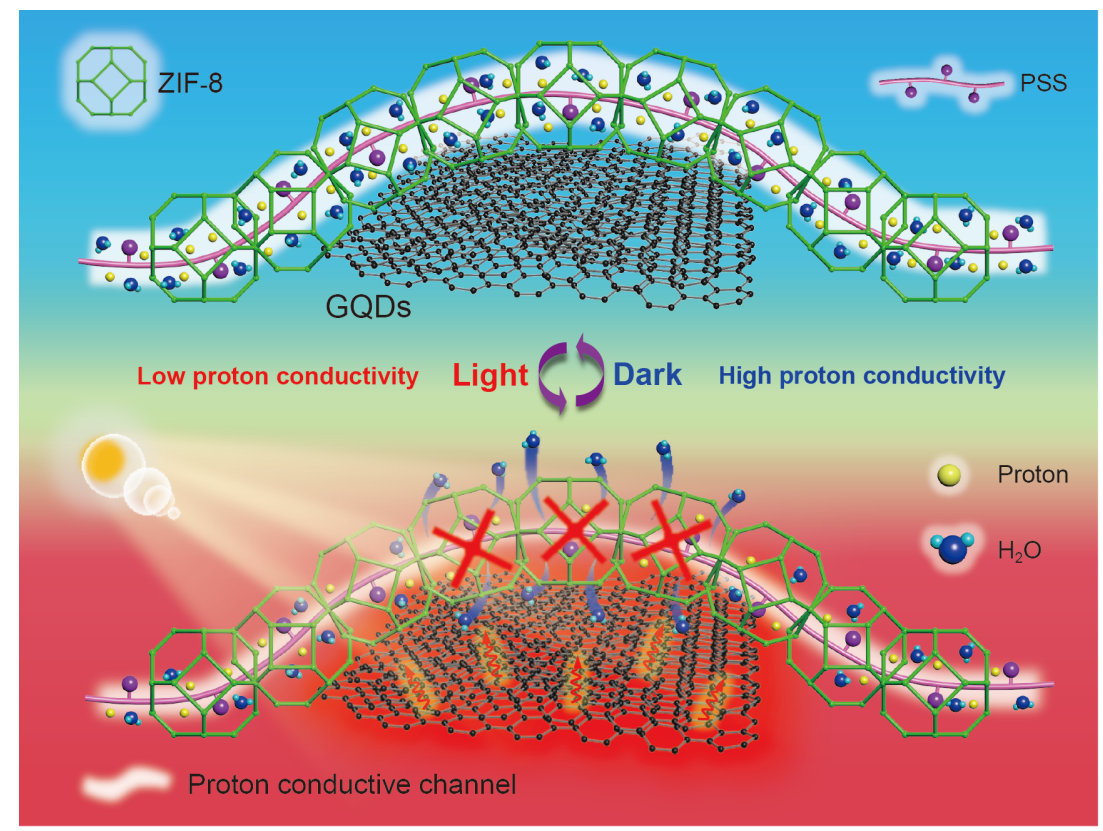

Scheme 2 Illustration of the proton transport mechanism through the photothermal effect of GQDs in the GQDs-PSS@ZIF-8 membranes.

the peaks of the GQD aqueous dispersion are not observed in the GQDs-PSS@ZIF-8 membranes, and the concentration of GQDs in the thin membrane is much higher than that in the aqueous dispersion. It is reasonable to imply that GQD particles generally have a strong tendency to undergo aggregation-induced emission quenching (so-called concentration quenching) in the condensed state, especially in the solid membrane, which causes significant energy loss through nonradiative relaxation processes [38]. Therefore, the concentration quenching of GQDs confined in ZIF-8 crystals takes place first in GQDs-PSS@ZIF-8 membranes instead of demonstrating fluorescence. This mechanism is another way to realize light-to-heat conversion. With increasing GQD loading, more heat is produced in the membranes, which corresponds to the decreasing PL intensity from 1GQDs-PSS@ZIF-8 to 5GQDs-PSS@ZIF-8. Moreover, the photothermal effect of GQDs also works in the visible light range, in which it shows broad absorption as a supplementary impact on heat generation. The PL lifetime curves are shown in Fig. S15, and the results of the fitting parameters of the PL lifetime decay measured for the membranes are provided in Table S3. The average lifetime (12.99 ns) of 2GQDs-PSS@ZIF-8 is shorter than the average lifetimes of PSS@ZIF-8 (15.12 ns) and ZIF-8 $(18.85 \mathrm{~ns})$. This result further confirms the fluorescence quenching mechanism mentioned above.

In addition, the good recovery of proton conductivity is another useful indicator for light-responsive devices. When turning off the light, water molecules return to the channels from the surroundings to strengthen the hydrogen-bond networks and supply proton carriers, significantly facilitating proton transport through the membranes. The system can reversibly maintain a balance in a humid environment with and without light. After being kept at room temperature for three months, the proton conductivity $\left(3.40 \times 10^{-5} \mathrm{~S} \mathrm{~cm}^{-1}\right)$ and ON/OFF ratio (12.6) of 2GQDs-PSS@ZIF-8 basically remain unchanged (Fig. S16) compared with those at the initial state, indicating the good stability of the membrane.

Infrared photos were taken to verify the heat generation in the samples when the lamp was cycled on and off at room temperature and with equal exposure time. From Fig. $4 \mathrm{e}, \mathrm{f}$, we can see that the average temperature of 2GQDs-PSS@ZIF-8 increases from 26.4 to $41.5^{\circ} \mathrm{C}$ after exposure to the Xenon lamp. The average temperature variations of the ZIF-8 and PSS@ZIF-8 membranes within the dotted line circles are lower than those of the GQDs-PSS@ZIF-8 membranes, whose surface colors are much brighter (Fig. S17). With an increasing GQD content, the surface temperatures of GQDs-PSS@ZIF-8 membranes increase in light, which matches the tendency of the PL spectra (Fig. 2b). This result confirms that the energy of the lamp light is converted into thermal energy, causing the GQDs-PSS@ZIF-8 membranes to increase in temperature. 


\section{CONCLUSIONS}

In conclusion, a light-controlled MOF-based membrane was synthesized by encapsulating PSS and GQDs into ZIF-8 crystals, whose proton conductivity can be modulated by light. PSS with hydrophilic sulfonate groups improves the proton conductivity of ZIF-8-based membranes by introducing more water molecules. GQDs act as stimuli-responsive materials in response to light. Upon illumination, the fluorescence quenching of ZIF-8 and the photothermal effect of GQDs endow the membranes with photoswitchable proton conductivity by local photothermal heating, which allows water molecules to escape from the proton transport channels and ultimately cuts off the proton conductive paths. The recovery process of proton conduction is due to regaining water molecules from the surroundings when without light. The 2GQDsPSS@ZIF-8 simply responds to light within minutes and possesses an $\mathrm{ON} / \mathrm{OFF}$ ratio of 12.8 at $55^{\circ} \mathrm{C}$ and $95 \% \mathrm{RH}$, which is much higher than that of pristine ZIF-8 (2.9). This study provides a new approach to the remote control of proton conduction by light in MOF-based protonic solids under hydrated conditions. The results show promise for application in photoswitchable devices.

\section{Received 3 November 2020; accepted 31 December 2020;} published online 1 March 2021

1 Kanj AB, Chandresh A, Gerwien A, et al. Proton-conduction photomodulation in spiropyran-functionalized MOFs with large on-off ratio. Chem Sci, 2020, 11: 1404-1410

2 Liang HQ, Guo Y, Shi Y, et al. A light-responsive metal-organic framework hybrid membrane with high on/off photoswitchable proton conductivity. Angew Chem, 2020, 132: 7806-7811

3 Amdursky N, Wang X, Meredith $\mathrm{P}$, et al. Long-range proton conduction across free-standing serum albumin mats. Adv Mater, 2016, 28: 2692-2698

4 Horike S, Umeyama D, Kitagawa S. Ion conductivity and transport by porous coordination polymers and metal-organic frameworks. Acc Chem Res, 2013, 46: 2376-2384

5 Ramaswamy P, Wong NE, Shimizu GKH. MOFs as proton conductors: Challenges and opportunities. Chem Soc Rev, 2014, 43: 5913-5932

6 Li PZ, Wang XJ, Liu J, et al. A triazole-containing metal-organic framework as a highly effective and substrate size-dependent catalyst for $\mathrm{CO}_{2}$ conversion. J Am Chem Soc, 2016, 138: 2142-2145

7 Wang Z, Cohen SM. Postsynthetic modification of metal-organic frameworks. Chem Soc Rev, 2009, 38: 1315-1329

8 He S, Wang $\mathrm{H}$, Zhang $\mathrm{C}$, et al. A generalizable method for the construction of MOF@polymer functional composites through surface-initiated atom transfer radical polymerization. Chem Sci, 2019, 10: 1816-1822

9 Guo Y, Peng X. Mass transport through metal organic framework membranes. Sci China Mater, 2018, 62: 25-42

10 Guo Y, Jiang Z, Ying W, et al. A DNA-threaded ZIF-8 membrane with high proton conductivity and low methanol permeability. Adv
Mater, 2017, 30: 1705155

11 Yamada T, Otsubo K, Makiura R, et al. Designer coordination polymers: Dimensional crossover architectures and proton conduction. Chem Soc Rev, 2013, 42: 6655-6669

12 Heinke L, Wöll C. Surface-mounted metal-organic frameworks: Crystalline and porous molecular assemblies for fundamental insights and advanced applications. Adv Mater, 2019, 31: 1806324

13 Nagarkar SS, Horike S, Itakura T, et al. Enhanced and optically switchable proton conductivity in a melting coordination polymer crystal. Angew Chem Int Ed, 2017, 56: 4976-4981

14 Saha R, Mukherjee PS. Chemistry of photoswitching molecules in the confined nanospace of aqueous molecular vessels. Dalton Trans, 2020, 49: 1716-1720

15 Müller K, Helfferich J, Zhao F, et al. Switching the proton conduction in nanoporous, crystalline materials by light. Adv Mater, 2018, 30: 1706551

16 Garg S, Schwartz H, Kozlowska M, et al. Conductance photoswitching of metal-organic frameworks with embedded spiropyran. Angew Chem Int Ed, 2019, 58: 1193-1197

17 Li P, Li Z, Guo Y, et al. Ag-DNA@ZIF-8 membrane: A proton conductive photoswitch. Appl Mater Today, 2020, 20: 100761

18 Zhou W, Gao X, Liu D, et al. Gold nanoparticles for in vitro diagnostics. Chem Rev, 2015, 115: 10575-10636

19 Boca SC, Potara M, Gabudean AM, et al. Chitosan-coated triangular silver nanoparticles as a novel class of biocompatible, highly effective photothermal transducers for in vitro cancer cell therapy. Cancer Lett, 2011, 311: 131-140

20 Xuan Y, Zhang RY, Zhang XS, et al. Targeting N-doped graphene quantum dot with high photothermal conversion efficiency for dual-mode imaging and therapy in vitro. Nanotechnology, 2018, 29: 355101

21 Tian Z, Yao X, Ma K, et al. Metal-organic framework/graphene quantum dot nanoparticles used for synergistic chemo- and photothermal therapy. ACS Omega, 2017, 2: 1249-1258

22 Li Z, Guo Y, Ying W, et al. Highly conductive and transparent metal-organic frameworks thin film. Sci China Mater, 2019, 62: 1350-1356

23 Yang X, Yan D. Direct white-light-emitting and near-infrared phosphorescence of zeolitic imidazolate framework-8. Chem Commun, 2017, 53: 1801-1804

24 Park KS, Ni Z, Côté AP, et al. Exceptional chemical and thermal stability of zeolitic imidazolate frameworks. Proc Natl Acad Sci USA, 2006, 103: 10186-10191

25 Barbosa P, Rosero-Navarro NC, Shi FN, et al. Protonic conductivity of nanocrystalline zeolitic imidazolate framework 8 . Electrochim Acta, 2015, 153: 19-27

26 Zhang R, Ji S, Wang N, et al. Coordination-driven in situ selfassembly strategy for the preparation of metal-organic framework hybrid membranes. Angew Chem Int Ed, 2014, 53: 9775-9779

27 Guo Y, Ying Y, Mao Y, et al. Polystyrene sulfonate threaded through a metal-organic framework membrane for fast and selective lithium-ion separation. Angew Chem Int Ed, 2016, 55: $15120-15124$

28 Biswal BP, Shinde DB, Pillai VK, et al. Stabilization of graphene quantum dots (GQDs) by encapsulation inside zeolitic imidazolate framework nanocrystals for photoluminescence tuning. Nanoscale, 2013, 5: 10556-10561

29 Mao Y, Li J, Cao W, et al. General incorporation of diverse components inside metal-organic framework thin films at room temperature. Nat Commun, 2014, 5: 5532 
30 Peng X, Jin J, Kobayashi N, et al. Time-dependent growth of zinc hydroxide nanostrands and their crystal structure. Chem Commun, 2008, 44: 1904-1906

31 Peng X, Jin J, Ichinose I. Mesoporous separation membranes of polymer-coated copper hydroxide nanostrands. Adv Funct Mater, 2007, 17: 1849-1855

32 Wang $\mathrm{Y}$, Wang $\mathrm{B}$, Shi $\mathrm{H}$, et al. Carbon nanodots in ZIF-8: Synthesis, tunable luminescence and temperature sensing. Inorg Chem Front, 2018, 5: 2739-2745

33 Agmon N. The Grotthuss mechanism. Chem Phys Lett, 1995, 244: 456-462

34 Sadakiyo M, Yamada T, Kitagawa H. Hydrated proton-conductive metal-organic frameworks. ChemPlusChem, 2016, 81: 691-701

35 Kreuer KD, Rabenau A, Weppner W. Vehicle mechanism, a new model for the interpretation of the conductivity of fast proton conductors. Angew Chem Int Ed Engl, 1982, 21: 208-209

36 Liu W, Yan X, Chen J, et al. Novel and high-performance asymmetric micro-supercapacitors based on graphene quantum dots and polyaniline nanofibers. Nanoscale, 2013, 5: 6053-6062

37 Zhu Z, Ma J, Wang Z, et al. Efficiency enhancement of perovskite solar cells through fast electron extraction: The role of graphene quantum dots. J Am Chem Soc, 2014, 136: 3760-3763

38 Lee J, Aizawa N, Numata M, et al. Versatile molecular functionalization for inhibiting concentration quenching of thermally activated delayed fluorescence. Adv Mater, 2017, 29: 1604856

Acknowledgements This work was supported by the National Natural Science Foundation of China (21875212), the Key Program of National Natural Science Foundation (51632008), the Major R\&D Plan of Zhejiang Natural Science Foundation (LD18E020001), the National Key Research and Development Program (2016YFA0200204) and the Fundamental Research Funds for the Central Universities.

Author contributions Peng X supervised the project; Fan S performed the experiments, analyzed the results and wrote the manuscript; Wang S, Hussain S and Li Z assisted in the device fabrication and measurement; Ma X, Wang X and Wan X contributed to the theoretical and data analyses. All authors contributed to the general discussion.

Conflict of interest The authors declare that they have no conflict of interest.

Supplementary information online version of the paper.

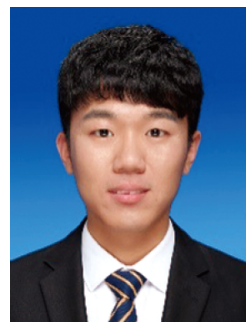

Shuaikang Fan received his Bachelor degree (2019) from the College of Materials Science and Engineering at Sichuan University. He is currently pursuing his Master degree at the School of Materials Science and Engineering at Zhejiang University under the supervision of Prof. Xinsheng Peng. His present research interests mainly focus on the design and syntheses of proton conductive MOF membranes for energy conversion devices.

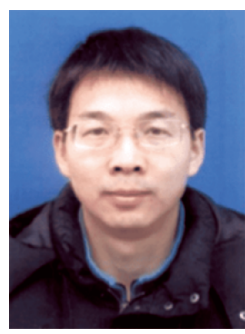

Xinsheng Peng received his $\mathrm{PhD}$ degree in 2003 at the Institute of Solid State Physics, Chinese Academy of Sciences. He became a full professor at the School of Materials Science and Engineering at Zhejiang University in 2010. His research interests mainly focus on the design and syntheses of functional membranes and controlled mass transportation in energy and environmental science.

\section{一种石墨烯量子点和聚苯乙烯磺酸共修饰的光控 质子传导ZIF-8薄膜}

范帅康, 王世林, 王小涁, 李卓异, 马旭, 万昕艺,

Shabab Hussain, 彭新生

摘要 可遥控的智能质子传导金属有机骨架(MOFs)薄膜在传感和 生物过程等领域具有诱人的应用前景. 本文通过固态限制转换方 法将聚苯乙烯磺酸(PSS)和石墨烯量子点(GQDs)共同封装到ZIF-8 中, 成功制备了具有光控质子传导特性的ZIF-8薄膜 (GQD PSS@ZIF-8). GQDs-PSS@ZIF-8膜的质子电导率是ZIF-8膜的6.3倍, 并且实现了其质子电导率的光可逆控制. 其机理主要是在苂光猝 灭和光热转换效应下光能被转换为热, 使膜局部温度升高, 水分子 从多孔通道中逸出, 从而切断质子传输路径, 导致质子电导率下降. 光照停止后, 水分子重新回到孔道中, 使质子传导率恢复. GQDsPSS@ZIF-8膜具有较好的光响应性能, 其开关比为 12.8. MOFs中的 光控质子传导性能对促进MOF基质子传导固体的发展及其在先进 光电设备中的应用具有潜在价值. 